\title{
Extrapolating the Fractal Characteristics of an Image Using Scale-Invariant Multiple-Point Statistics
}

\author{
Grégoire Mariethoz • Philippe Renard • \\ Julien Straubhaar
}

Received: 19 December 2010 / Accepted: 27 August 2011 / Published online: 5 October 2011 (C) International Association for Mathematical Geosciences 2011

\begin{abstract}
The resolution of measurement devices can be insufficient for certain purposes. We propose to stochastically simulate spatial features at scales smaller than the measurement resolution. This is accomplished using multiple-point geostatistical simulation (direct sampling in the present case) to interpolate values at the target scale. These structures are inferred using hypothesis of scale invariance and stationarity on the spatial patterns found at the coarse scale. The proposed multiple-point super-resolution mapping method is able to deal with "both continuous and categorical variables," and can be extended to multivariate problems. The advantages and limitations of the approach are illustrated with examples from satellite imaging.
\end{abstract}

Keywords Geostatistics · Multiple-point · Zooming $\cdot$ Resolution $\cdot$ Scale invariance Fractal

\section{Introduction}

High-resolution models are needed to describe and predict large- and small-scale physical phenomena. Such fine models are much needed in various fields of Earth Science, such as for example hydrology, hydrogeology and meteorology (Von Storch et al. 1993; Wilby and Wigley 1997; Ferraris et al. 2003; McCabe and Wood 2006; Liu et al. 2008).

G. Mariethoz $(\varangle)$

National Centre for Groundwater Research and Training, University of New South Wales, Sydney, Australia

e-mail: gregoire.mariethoz@minds.ch

G. Mariethoz $\cdot$ P. Renard · J. Straubhaar

Centre for Hydrogeology, University of Neuchâtel, 11 Rue Emile Argand, CP 158, 2000 Neuchâtel, Switzerland 
Most models require input parameters coming from various kinds of measurement device whose sampling resolution is limited (Bertero and Boccacci 2003). Hence, the measurement scale is often larger than the scale at which physical processes occur, making the understanding of small-scale phenomena a challenging issue, even when important datasets are available (Schulze-Makuch and Cherkauer 1998; Zlotnik et al. 2000). This situation is not likely to change because no matter the resolution of the data, some limitation on the measurement scale will always remain. Characterizing small-scale structures is critical for a number of applications. For example, small-scale heterogeneity has a major impact on the permeability of heterogeneous subsurface media (Sánchez-Vila et al. 1996; Wen and Gomez-Hernandez 1998; Harter 2005). The same problem occurs when one needs to estimate rainfall when radar measurements only provide a coarse representation of the phenomenon (Lovejoy and Mandelbrot 1985; Lovejoy and Schertzer 1990; Marsan et al. 1996).

The problem treated in the present paper, often known as image zooming or superresolution mapping, is the case where the variable of interest is exhaustively known, but the resolution of this information is too low. There is a missing scale where no information is available. Since information at a scale smaller than the measurement scale is not available in the data, it has to be inferred using assumptions on the target scale (Baker and Kanade 2002). Super-resolution mapping has been addressed in the image processing literature and successfully applied to digital photography. In this paper, we present an alternative technique based on multiple-point geostatistics and apply it to remote sensing images. In image processing, a number of methods and algorithms are devoted to super-resolution mapping (see a review in Farsiu et al. 2004). Excellent results are given by example-based techniques using training images (TI) (Atkins et al. 1999; Freeman et al. 2002) that derive the relationship between small-scale features and their coarse counterparts from a database of highresolution TIs and their coarsened/blurred versions. Other methods (Tsai and Huang 1984; Sroubek et al. 2007) fuse a sequence of low-resolution images (for example successive frames of a movie scene) to produce a higher-resolution image.

Image processing techniques also offer several tools for edge detection and enhancement to achieve visually appealing results (Fattal 2007; Wang and Gong 2008; Dai et al. 2009). In Earth Sciences, however, it is often difficult to use such visual assumptions that cannot be validated in the absence of data at small scale. Moreover, edges are characteristics determining the interpretation of an image by a human eye, whereas for Earth Science models, it is rather concepts of heterogeneity and connectivity that are the most influential on the model outcomes. For hydrogeological models where the variable of interest is hydraulic conductivity, efficient superresolution mapping can be performed through an inverse procedure (Wen et al. 1997; Grimstad et al. 2003) relying on indirect data such as for example production history from wells or pumping tests data. Such problems, often known as downscaling, include physical relationships to constrain the small-scale spatial structure. For example, when downscaling hydraulic conductivity, the local equivalent (upscaled) conductivity of a downscaled area must be equal to the respective coarse-scale values (Renard and de Marsily 1997). In the context of geostatistics, techniques such as block simulation (Liu and Journel 2008) are available to account for these constraints when the relation between the variables can be expressed as rather simple analytical 
expressions. Such methods are efficient for specific hydrogeological problems where data are available as a base for the inverse procedure. Sometimes the spatial structure at the small scale can be deduced from analogous sites (or proxys) whose small-scale spatial structure is known. Corresponding spatial statistics can be represented using geostatistical tools such as variograms. For example, in problems involving land cover estimation based on coarse remote sensing images, Nguyen et al. (2006) and Tatem et al. (2002) use super-resolution mapping to estimate the class composition of the coarse pixels as well as the spatial distribution of these classes within the pixels. Super-resolution mapping is formulated as an optimization problem that is solved using a Hopfield neural network. Constraints given by variograms derived from proxys are imposed on the spatial structures at sub-pixel scale.

Another representation of spatial statistics is given by multiple-point (MP) geostatistical methods (Guardiano and Srivastava 1993). Similarly to image processing techniques, they use the concept of a training image as a prior model representing the spatial structure of a given variable (Journel and Zhang 2006). These methods are able to reproduce high-order spatial statistics, thus exploiting complex heterogeneous structures present in the TI. Several variants of multiple-point simulation are found in the literature, with certain methods adapted for categorical and/or continuous variables (Guardiano and Srivastava 1993; Strebelle 2002; Arpat and Caers 2007; El Ouassini et al. 2008; Wu et al. 2008; Remy et al. 2009; Mariethoz et al. 2010; Straubhaar et al. 2011), and some that are parallelizable (Straubhaar et al. 2008; Mariethoz 2010). A stochastic formulation of super-resolution mapping using multiplepoint geostatistical methods was investigated by Boucher et al. (2008). It allows inferring the small-scale heterogeneity using a proxy, but finding an adequate proxy is often difficult, making the method sometimes impractical. Although our approach also uses multiple-point statistics, we circumvent the problem of finding a TI by using the multiple-point statistics of the coarse image, under the assumption of scale invariance.

Despite numerous existing techniques, there is still a need for generic superresolution algorithms capable of producing satisfactory high-resolution images using one coarse image only (as opposed to methods using additional information to inform the fine scale, such as TIs, series of coarse images or statistics derived from a proxy). We address the super-resolution problem for cases where edges delineation is not the critical validation criterion, where no indirect data are available that can constrain the inference of small-scale spatial variability using physical relationships, and where no proxy cases are available. We propose to use multiple-point stochastic simulation tools not to reproduce the patterns found in a TI, but to propagate the high-order spatial statistics from the coarse scale to the fine scale. Hence the only input needed for modeling patterns at the small scale is the fully informed pattern at a coarse scale, and an assumption of scale invariance allowing for transferring coarse scale features to a finer scale. The key idea is to perform super-resolution using multifractal cascades (Mandelbrot 1974) combined with MP simulations for reconstructing the fine-scale structures. The specificity of our approach is that the algorithm does not need a TI or a proxy, because we directly infer MP statistics from the coarse image and we use them at the finer target scale. This algorithm is straightforward in terms of implementation and parameterization, and is robust as long as scale-invariant features are 
concerned. Moreover, implemented in a stochastic framework, it allows for a probabilistic solution for image zooming problems.

The first part of the paper presents the direct sampling (DS) algorithm, which is the main tool for solving the super-resolution mapping problems. The second section describes the super-resolution algorithm and presents illustrative examples.

\section{The Direct Sampling Simulation Method}

Direct sampling is a geostatistical method based on multiple-point statistics (Mariethoz and Renard 2010; Mariethoz et al. 2010). It aims at producing realizations of a spatially dependant variable $Z$ at all $N$ locations $\mathbf{x}_{i}$ of a regular grid, with $i=[1, \ldots, N]$. These locations can be assimilated to the pixels of an image. Since $Z$ is spatially structured, its value at each location $Z(\mathbf{x})$ depends on the values of its spatial neighbors. The principle of all multiple-point methods is to infer this dependency from a TI. $Z(\mathbf{x})$ can be either a continuous or categorical variable. Note that although we adopt the notation for continuous variables, all concepts presented in this paper are also valid for the categorical case.

A realization of $Z$ should have a similar spatial dependence as the TI, i.e., the relationship between the value at any location and all other values at all other locations. In order to respect all dependencies, each realization must be a sample of the $N$-dimensional joint distribution

$$
\mathrm{F}(\mathbf{x})=\mathrm{P}\left\{Z\left(\mathbf{x}_{1}\right), Z\left(\mathbf{x}_{2}\right), \ldots, Z\left(\mathbf{x}_{N}\right)\right\} .
$$

This can be accomplished this by assigning values to all locations using the sequential simulation paradigm (Deutsch and Journel 1992) which considers the following decomposition

$$
\mathrm{F}(\mathbf{x})=\mathrm{P}\left\{Z\left(\mathbf{x}_{1}\right)\right\} \cdot \mathrm{P}\left\{Z\left(\mathbf{x}_{2}\right) \mid z\left(\mathbf{x}_{1}\right)\right\} \cdots \cdot \mathrm{P}\left\{Z\left(\mathbf{x}_{m}\right) \mid z\left(\mathbf{x}_{1}\right), \ldots, z\left(\mathbf{x}_{m-1}\right)\right\} .
$$

The principle is that each location $\mathbf{x}$ in the grid is visited in a random order (usually referred to as simulation path). The value of $Z(\mathbf{x})$ is then computed accounting for the spatial dependence between the values at all neighboring locations. The values of those neighboring locations are already known either because they have been simulated previously in the sequential simulation, or because they correspond to measurements taken at these locations. Once the value of $Z(\mathbf{x})$ is determined, it is considered known and used to condition the distribution of possible attribute values at locations that are visited thereafter. The result, according to (2), is that the outcome at each location is conditional to all previously simulated pixels values.

A common approximation is to consider only a limited neighborhood of size $n$, with $n \ll N$ to limit computational burden. The lag vectors $\mathbf{L}=\left\{\mathbf{h}_{1}, \ldots, \mathbf{h}_{n}\right\}=$ $\left\{\mathbf{x}_{1}-\mathbf{x}, \ldots, \mathbf{x}_{n}-\mathbf{x}\right\}$ define the locations of the neighbors of $\mathbf{x}$, denoted $\mathbf{x}_{1}, \mathbf{x}_{1}, \ldots, \mathbf{x}_{n}$. The data event centered on $\mathbf{x}$ is the combination of the lag vectors and the values at the locations pointed by the lag vectors $\mathbf{d}_{n}(\mathbf{x}, \mathbf{L})=\left\{Z\left(\mathbf{x}+\mathbf{h}_{1}\right), \ldots, Z\left(\mathbf{x}+\mathbf{h}_{n}\right)\right\}$. Given this data event, the decomposition in (2) calls for obtaining a single sample of the conditional probability distribution function (cpdf)

$$
\mathrm{F}\left(\mathbf{z}, \mathbf{x}, \mathbf{d}_{n}\right)=\operatorname{Prob}\left\{Z(\mathbf{x}) \leq z \mid \mathbf{d}_{n}(\mathbf{x}, \mathbf{L})\right\} .
$$


The sample from (3) is assigned to $Z(\mathbf{x})$, which is thereafter considered as conditioning data when simulating the remaining nodes. At each visited node $\mathbf{x}$, the direct sampling algorithm generates a sample of (3) by the following steps (Shannon 1948; Mariethoz et al. 2010), as follows:

Given a pattern $\mathbf{d}_{n}(\mathbf{x}, \mathbf{L})$ in the simulation grid:

1. Define distance threshold $t \in[0,1]$.

2. Iterate until a stopping criterion is met:

(a) Select randomly a location $\mathbf{y}$ in the training image.

(b) Determine $\mathbf{d}_{n}(\mathbf{y}, \mathbf{L})$, the data event centered on $\mathbf{y}$.

(c) Compute the distance $d\left\{\mathbf{d}_{n}(\mathbf{x}, \mathbf{L}), \mathbf{d}_{n}(\mathbf{y}, \mathbf{L})\right\}$.

(d) If the distance $d\left\{\mathbf{d}_{n}(\mathbf{x}, \mathbf{L}), \mathbf{d}_{n}(\mathbf{y}, \mathbf{L})\right\} \leq t$, assign $Z(\mathbf{x})=Z(\mathbf{y})$. Otherwise, try another location $\mathbf{y}$.

The term $d\left\{\mathbf{d}_{n}(\mathbf{x}, \mathbf{L}), \mathbf{d}_{n}(\mathbf{y}, \mathbf{L})\right\}$ represents the distance between two data events, one found in the simulation grid and the other one found in the training image. Using different measures of distance offers a high degree of flexibility. It is therefore possible to accommodate continuous and categorical variables, as well as multivariate cases. For categorical variables, the distance between a data event found in the simulation and another one found in the TI $d\left\{\mathbf{d}_{n}(\mathbf{x}, \mathbf{L}), \mathbf{d}_{n}(\mathbf{y}, \mathbf{L})\right\}$ is given by the proportion of non-matching nodes. It is calculated using the indicator variable $a$, which equals 0 if two nodes have identical value and 1 otherwise

$$
d\left\{\mathbf{d}_{n}(\mathbf{x}), \mathbf{d}_{n}(\mathbf{y})\right\}=\frac{1}{n} \sum_{i=1}^{n} a_{i} \in[0,1], \quad \text { where } a_{i}= \begin{cases}0 & \text { if } Z\left(\mathbf{x}_{i}\right)=Z\left(\mathbf{y}_{i}\right) \\ 1 & \text { if } Z\left(\mathbf{x}_{i}\right) \neq Z\left(\mathbf{y}_{i}\right) .\end{cases}
$$

A convenient measure of distance able to accommodate continuous variables is the normalized pair wise Manhattan distance

$$
d\left\{\mathbf{d}_{n}(\mathbf{x}), \mathbf{d}_{n}(\mathbf{y})\right\}=\frac{1}{n} \sum_{i=1}^{n} \frac{\left|Z\left(\mathbf{x}_{i}\right)-Z\left(\mathbf{y}_{i}\right)\right|}{\operatorname{argmax}\left(Z^{t i}\right)-\operatorname{argmin}\left(Z^{t i}\right)} \in[0,1] .
$$

In case the data event is multivariate, with $m$ variables $Z_{k}, k=1, \ldots, m, Z(\mathbf{x})$ needs to be a sample of the ccdf

$$
F_{k}\left(z, \mathbf{x}, \mathbf{d}_{n_{1}}^{1}, \ldots, \mathbf{d}_{n_{m}}^{m}\right)=\operatorname{Prob}\left\{Z_{k}(\mathbf{x}) \leq z \mid \mathbf{d}_{n_{1}}^{1}\left(\mathbf{x}, \mathbf{L}^{1}\right), \ldots, \mathbf{d}_{n_{m}}^{m}\left(\mathbf{x}, \mathbf{L}^{m}\right)\right\} .
$$

Note that each variable $Z_{k}$ can have a specific data event $\mathbf{d}_{n_{k}}^{k}\left(\mathbf{x}, \mathbf{L}^{k}\right)=$ $\left\{Z_{k}\left(\mathbf{x}+\mathbf{h}_{1}^{k}\right), \ldots, Z_{k}\left(\mathbf{x}+\mathbf{h}_{n_{k}}^{k}\right)\right\}$. The distance between a joint data event found in the simulation and one found in the TI is then defined as a weighted average of the individual distances defined previously,

$$
\begin{aligned}
& d\left\{\mathbf{d}_{n}(\mathbf{x}), \mathbf{d}_{n}(\mathbf{y})\right\}=\sum_{k=1}^{m} w_{k} d\left\{\mathbf{d}_{n_{k}}^{k}\left(\mathbf{x}, \mathbf{L}^{k}\right), \mathbf{d}_{n_{k}}^{k}\left(\mathbf{y}, \mathbf{L}^{k}\right)\right\} \in[0,1], \\
& \quad \text { with } \sum_{k=1}^{m} w_{k}=1 \text { and } w_{k} \geq 0 .
\end{aligned}
$$


The weights $w_{k}$ are defined by the user. Setting different weights allows accounting for the fact that the pertinent measure of distance may be different for each variable. Joint simulations are performed using a single (random) path that visits all components of vector $Z$ at all nodes of the simulation grid.

\section{Dealing with a Missing Scale}

We propose a general super-resolution method that does not rely on any other input data than a single coarse image (i.e., it does not need an external training image) and that has the advantage of being very parsimonious in parameterization. The central idea is to use the known coarse image as a training image for inferring small-scale patterns. It assumes that the parameters of interest have properties of scale invariance (Mandelbrot 1967), thus allowing to infer the fine scale structures from the coarse ones. This assumption constitutes a model for the missing scale. The outcomes of physical processes can often present fractal properties. For example, self-similar characteristics have been observed for a wide range of geological physical properties (Kiraly 1988; Barton and La Pointe 1991; Turcotte 1992). Capitalizing on these fractal properties, it becomes possible to perform realistic super-resolution mapping of coarse images.

We propose to accomplish super-resolution mapping in a similar way as the fractal cascades proposed by Mandelbrot (1974). The main idea is to consider a coarse image as a sampling of the same image in high resolution. At each step of a two-dimensional super-resolution problem, the coarse nodes are divided in four children nodes. The value of each coarse node is assigned to one of its children. Next, we define a migration scheme that systematically attributes the value of the parent node to its the top left child node. Once this migration has been accomplished, all values of $Z$ are determined by multiple-point simulation. Where multiple-point simulation methods determine the value of the simulated pixels by using an external training image, we use instead the coarse image at its original resolution (all pixels are four times larger on the coarse image). The result is that the large-scale patterns of the coarse image are reproduced at the smaller simulation scale, resulting in scale-invariant structures. Treating a three-dimensional problem is identical, except that each coarse grid node has eight children nodes. Note that by migrating the value of coarse pixels at a small scale, we ignore possible scale and support effects in the variable of interest. In contrast to most downscaling techniques, this algorithm does not ensure that the mean of children nodes values is equal to the integral value of their respective parent nodes.

In this paper, we use direct sampling simulation, but any other multiple-point method can be applied. Using traditional MP techniques instead of DS would call for considering the multiple-grids approach (Strebelle 2002), which would consist of creating an additional fine multiple-grid and assuming that the patterns catalogue (the search tree or the data events list) remains unchanged between this finer grid and the coarser ones. Nevertheless, problems would arise because of the migration of a large number of conditioning data between multiple-grids (Strebelle 2002). Moreover, super-resolution would be limited to categorical variables.

The super-resolution mapping algorithm proceeds as follows: 
a)

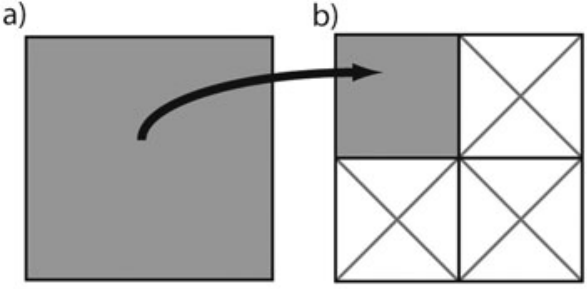

d)

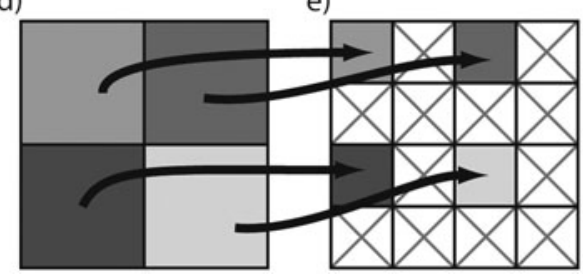

c)

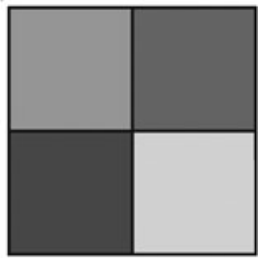

f)

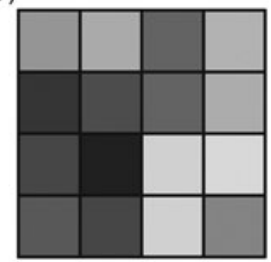

Fig. 1 Visual representation of the zooming procedure for a single coarse grid node with the deterministic migration scheme. (a) and (b) Migrate the value of the coarse grid to the top left one of the 4 possible equivalent fine grid nodes. (c) Simulate the remaining 3 fine grid nodes using the coarse grid as training image. (d), (e) and (f) Second super-resolution iteration

1. Given a coarse image on grid G1 of size $[X, Y]$, create a finer grid $\mathrm{G} 2$ of size $[2 X, 2 Y]$.

2. For each node $\mathbf{x}^{\mathrm{G} 1}$ of $\mathrm{G} 1$, assign $Z\left(\mathbf{x}^{\mathrm{G} 1}\right)$ to one of its 4 children nodes on $\mathrm{G} 2$.

3. Define a random path through all nodes of $\mathrm{G} 2$.

4. Simulate the remaining children nodes $Z\left(\mathbf{x}^{\mathrm{G} 2}\right)$ by multiple-point technique, using G1 as training image grid.

This procedure can be performed many times, using G2 as coarse grid and G4 (size $[4 X, 4 Y])$ as fine grid, etc., by iteratively applying a factor 2 . Figure 1 illustrates two iterations of super-resolution for a single coarse grid node.

Two major assumptions underlie our super-resolution mapping algorithm. The first one is the assumption of stationarity which is common to most geostatistical methods. It requires that the patterns found in a subset of the image are similar to the patterns found in another subset of the image. This assumption is required because multiplepoint methods extrapolate statistical properties to unknown locations at the fine scale, and can only do so if the attribute values in the part of the domain to complete are statistically equivalent to the part that is known. The second assumption particular to our approach is that the patterns at coarse and fine scales are compatible, i.e. that it is possible to populate the children nodes with rescaled coarse-scale patterns without causing incompatibilities. This assumption means that our method applies to images that have scale-invariant properties.

A possibility to extend the algorithm is to consider refinement factors greater than 2. For example, one can divide a pixel into nine (instead of four) pixels in twodimensions using a refinement factor of 3 in each direction. In this case, eight fine pixels would need to be simulated for each coarse pixel. Similarly, one can use a refinement factor of 5, or even more. The rest of the approach remains identical: the coarse scale value is associated to one pixel and the remaining unknown pixels are 
simulated using the multiple-point technique. Although this approach seems appealing because it can use refinement factors that are not multiples of 2 , it introduces artifacts due to the low density of data in the simulation grid. In the next section, we investigate both approaches, and show that successive refinement by a factor 2 is preferable because it does not introduce as much artifacts.

\section{Fractal Dimensions with a Categorical Variable}

Computation of fractal dimension is the standard way to quantify scale invariance. It is determined by computing the number of self-similar objects that are needed to cover an entire system as a function of the object size. A classical example of fractal dimension computation is the measurement of the length of a coastline using rulers of different sizes (Mandelbrot 1967). Smaller rulers results in longer coastlines because it takes into account more irregularities. If plotting on a log-log scale the coastline length as a function of the ruler size results in a linear function, the system can be termed self-similar on the corresponding range of scales (Ronayne and Gorelick 2006). The fractal dimension is then the slope of this line.

In order to use this measure to validate our method, we perform super-resolution simulation of a categorical image that has similar characteristics as a coastline. Fractal dimensions are then computed on both coarse and refined images, and we show that the refined image is a fractal extrapolation of the coarse one. The original coarse image (Fig. 2a) was obtained by segmentation of a satellite image (Lena delta, Russian Federation) in three categories representing rivers, land and lakes. Direct Sampling is used with the distance formulated in (4) to perform three super-resolution steps, each time using a factor 2 (respectively, corresponding to G2, G4 and G8. Figure 2b shows the resulting image refined once and Fig. 2c displays the image refined twice. The zoomed areas allow a detailed comparison of the small-scale structures. Where the coarse image shows stair-like interfaces between the different categories, the fine image is smoother, but still presents some degree of roughness. At the fine scale, the irregularities of the boundaries between facies reflect the features that occur at a larger scale in the coarse image. Note that on Fig. 2, the resolution increase is most visible between the coarse and the medium images. However, the fractal dimension analysis shows that a coherent zooming is performed at the fine scale as well.

A fractal dimension analysis of facies boundaries is performed on both images. The boundary of each category is delineated using an edge detection algorithm (Shih 2009) and the lengths of these boundaries are computed using rulers of different sizes. The resulting functions, plotted in a log-log scale, are shown in Fig. 3. With rulers sizes ranging from 1 to 12 (the unit length is one coarse pixel), the coarse and refined images have similar scale dependence for the boundary length of all 3 categories. A scale-invariant behavior, characterized by an almost linear function, is observed with ruler sizes between 1 and 5. This linear behavior is an indication that it is reasonable to make a hypothesis of scale invariance in this case. We perform the same boundary length analysis on the refined images, but since they have smaller pixels, it is also possible to measure boundary length with rulers of size $0.5,0.25$ and 0.125 , respectively for the first (G2), second (G4) and third (G8) degrees of refinement. The 

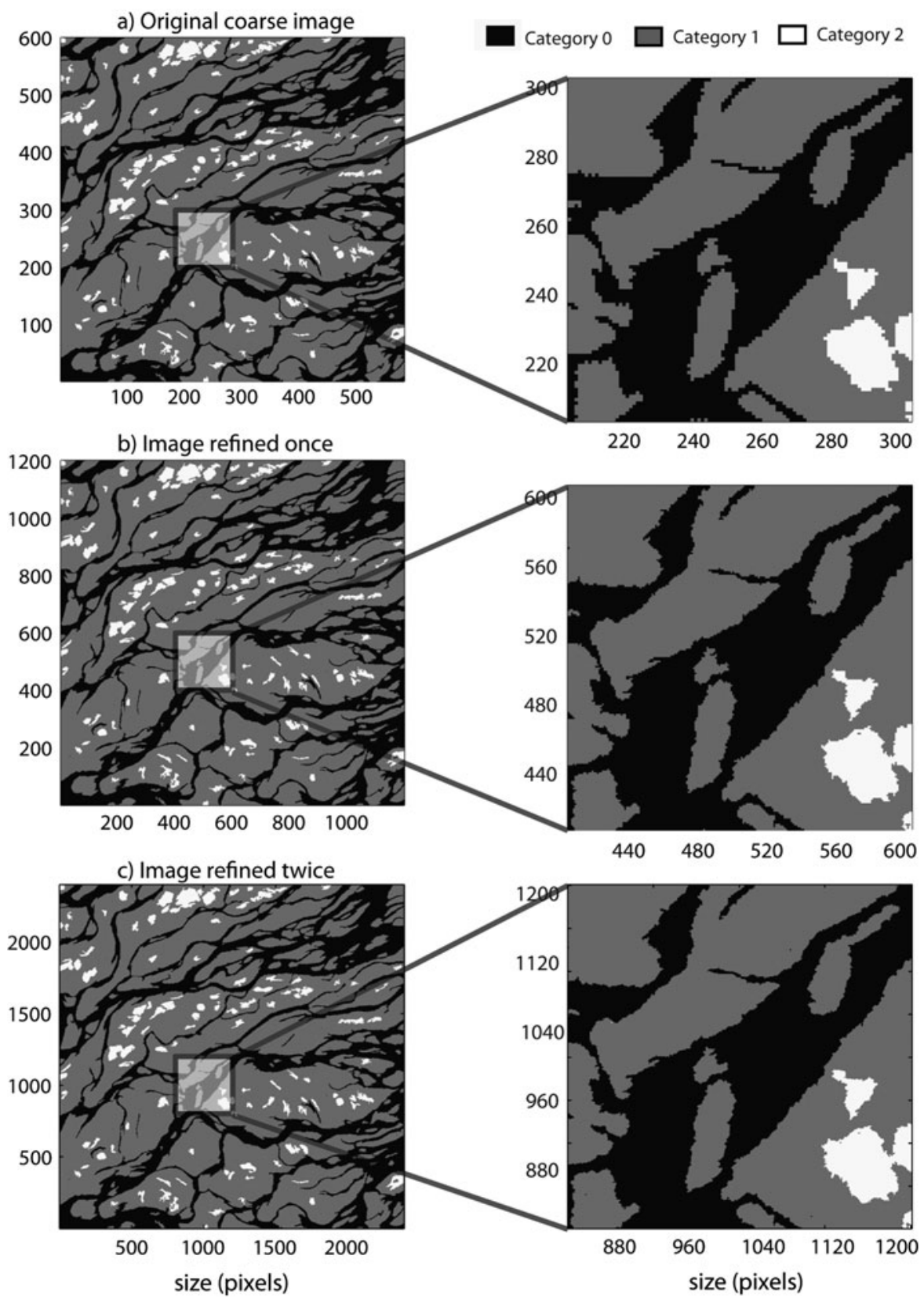

Fig. 2 Super-resolution of a Landsat image (USGS/EROS) of the Lena delta (Russian Federation) consisting of 3 categories. (a) Original coarse image. (b) Result after one super-resolution pass. (c) Result after two super-resolution passes 


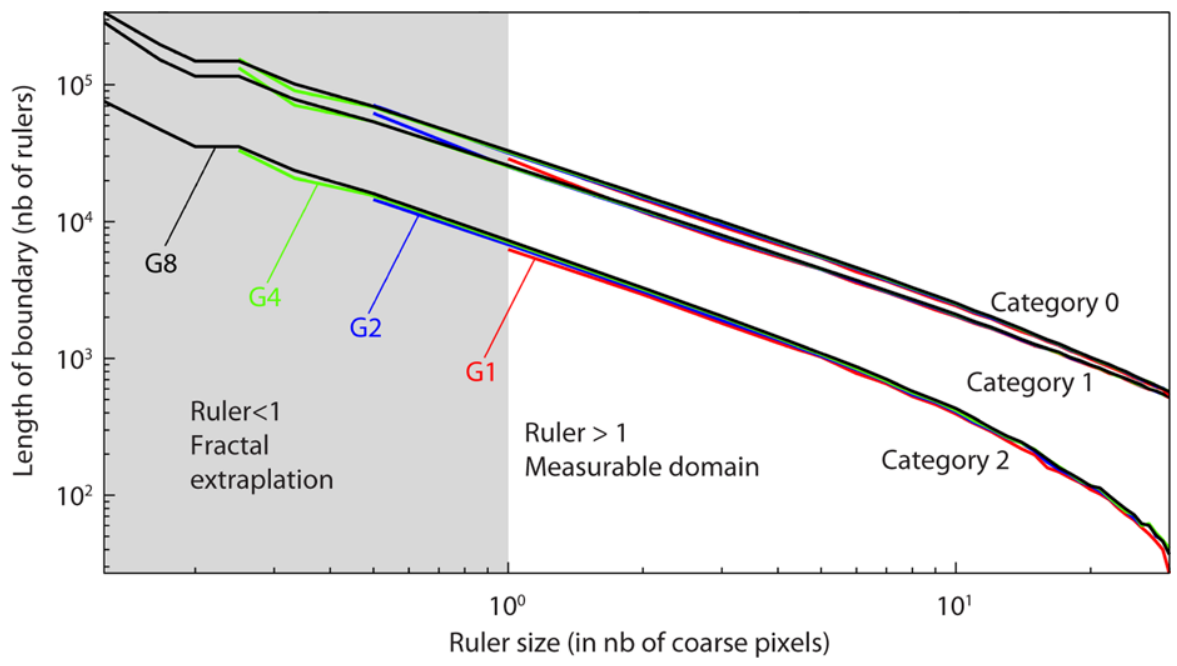

Fig. 3 Length of the boundary of each category measured with rulers of varying size with repeated application of a refinement factor 2 (length unit: one coarse pixel)

Table 1 Fractal dimension of the boundary length of each category, for original and images refined successively with a factor 2

\begin{tabular}{llll}
\hline & Category 0 & Category 1 & Category 2 \\
\hline Original coarse image (G1) & 1.1304 & 1.1201 & 1.2025 \\
Image refined once (G2) & 1.1145 & 1.1106 & 1.1912 \\
Image refined twice (G4) & 1.1045 & 1.1065 & 1.1844 \\
Image refined 3 times (G8) & 1.1004 & 1.1058 & 1.1774 \\
\hline
\end{tabular}

resulting boundary lengths are in the continuity of the linear function observed for larger ruler sizes, hence the fractal dimension of the coarse image is preserved.

Table 1 provides numerical values for the calculated fractal dimensions. Values for coarse and refined images are similar, showing that in this case, the method allows inferring small-scale features while preserving scale-invariant characteristics of the image. In Fig. 4, we display similar results using refinement factors larger than 2 . The method has been applied with refinement values ranging between 2 and 12. We see that the use of high refinement factors induces a bias of the fractal dimension which tends to increase (the slope increases) in this example, as shown in Table 2. We interpret this artificial increase in the fractal dimension as a consequence of noise, which is due to the large ratio of the number of nodes to be simulated versus the number of conditioning data. We conclude from this analysis that a refinement factor of 2 provides the most natural extrapolation of the image at a small scale as it preserves its fractal dimension. 


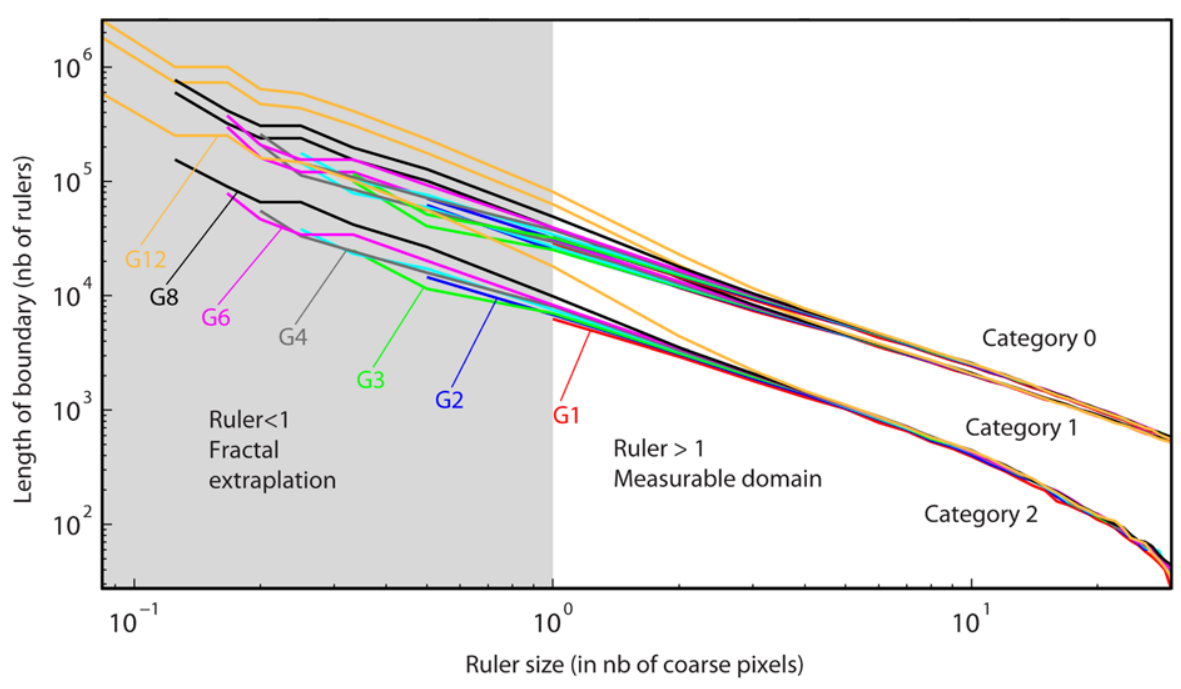

Fig. 4 Length of the boundary of each category measured with rulers of varying size, when applying refinement factors varying from 2 (G2) to 12 (G12) (length unit: one coarse pixel)

Table 2 Fractal dimension of the boundary length of each category, for original and images refined with factors larger than 2

\begin{tabular}{llll}
\hline & Category 0 & Category 1 & Category 2 \\
\hline Original coarse image (G1) & 1.1304 & 1.1201 & 1.2025 \\
Refinement factor 3 (G3) & 1.0725 & 1.0757 & 1.1511 \\
Refinement factor 4 (G4) & 1.1128 & 1.1228 & 1.1961 \\
Refinement factor 6 (G6) & 1.1610 & 1.1731 & 1.227 \\
Refinement factor 8 (G8) & 1.2728 & 1.2858 & 1.3264 \\
Refinement factor 12(G12) & 1.4370 & 1.4653 & 1.5484 \\
\hline
\end{tabular}

\section{Continuous Variable}

The next example illustrates our super-resolution mapping method applied to a continuous image that was built by applying geostatistical methods on an aerial photograph of braided channels in the Ohau River, New Zealand (Mosley 1982). The result is a grid populated with complex patterns of hydraulic log-transmissivity values $(T)$, showing heterogeneity and anisotropy. Kerrou et al. (2008) explain in detail the procedure used for generating the image. The image size is $1000 \mathrm{~m}$ by $400 \mathrm{~m}$ and it has been discretized in $440 \times 176$ grid nodes.

Two super-resolution steps are accomplished with the DS algorithm, using the measure of distance in (5) because the variable is continuous. Figure 5a shows the original coarse image G1. Figure 5 b depicts a detail of G1, with coarse pixels clearly visible. The super-resolution is performed many times, using each simulation from the previous step as a new coarse image that is sampled again. Figures $5 \mathrm{c}$ to $5 \mathrm{f}$ display the results of two super-resolution iterations (G2 and G4). In the detailed zone, the image becomes sharper at each step while preserving the structures present in 


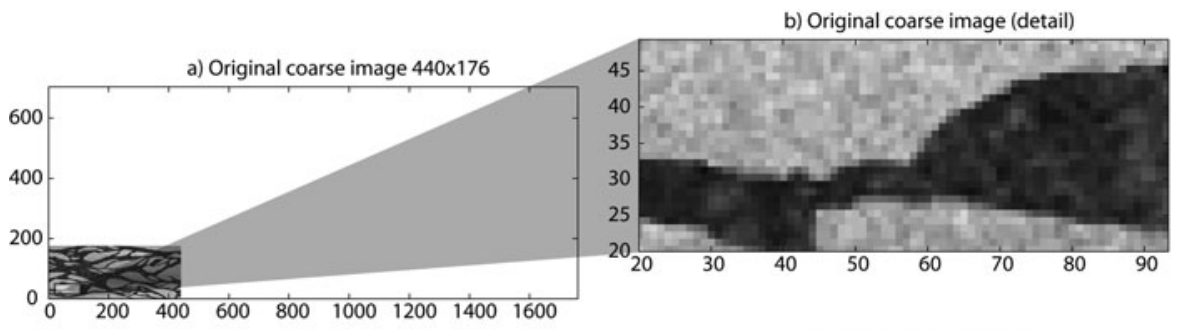

d) Medium image (detail)

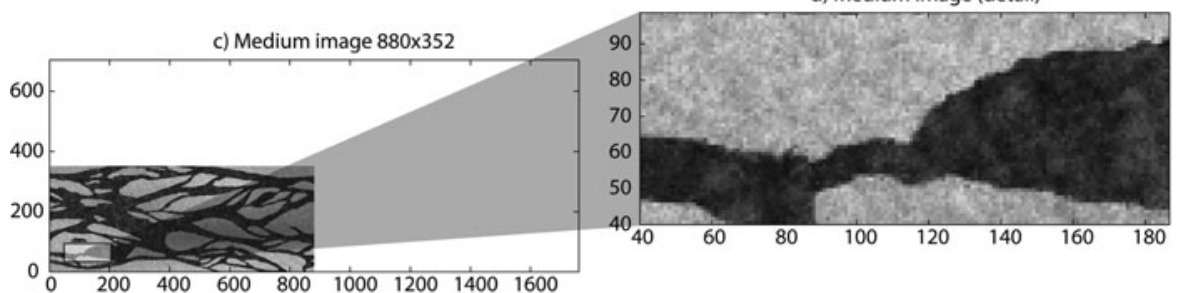

f) Fine image (detail)

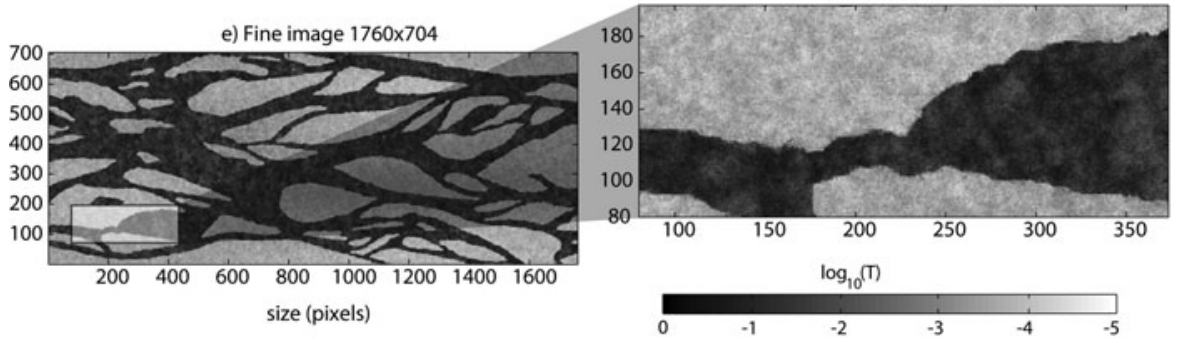

Fig. 5 Results of two super-resolution steps for a continuous variable. (a) Original coarse image (G1), which contains the only available input data for the super-resolution algorithm. (b) A detail of the coarse image. (c) Resulting image after one super-resolution iteration (G2). (d) A detail of the corresponding image. (e) Resulting image after two super-resolution iterations (G3). (f) A detail of the corresponding image. Note that the axes do not represent the real system of coordinates, but the number of nodes in each image

the coarse image. Comparison of the histograms of the three images (Fig. 6a) shows that super-resolution does not induce a bias in the distribution of transmissivity values. The variograms (Fig. 6b) clearly indicate that the spatial structure of the refined field is not affected. Histograms and variograms are perfectly reproduced, without performing any histogram transform or variogram adjustment.

\section{Conclusions}

In this paper, we present a method for performing super-resolution mapping or zooming of coarse images. Our method infers information on the missing scale by assuming fractal properties for the variable to be simulated. It proceeds by first migrating pixel values on a refined grid and in a second step by populating the rest of the fine grid with spatial patterns borrowed from the coarse image. For this second step, we use multiple-point geostatistical simulation methods (MPS). The main idea is to treat 
Fig. 6 (a) Histograms of the values of coarse, medium and fine images. (b) Omnidirectional variograms of the three images. Variograms lags have been computed using the real system of coordinates with a domain size of $1000 \times 400$ meters in the three cases
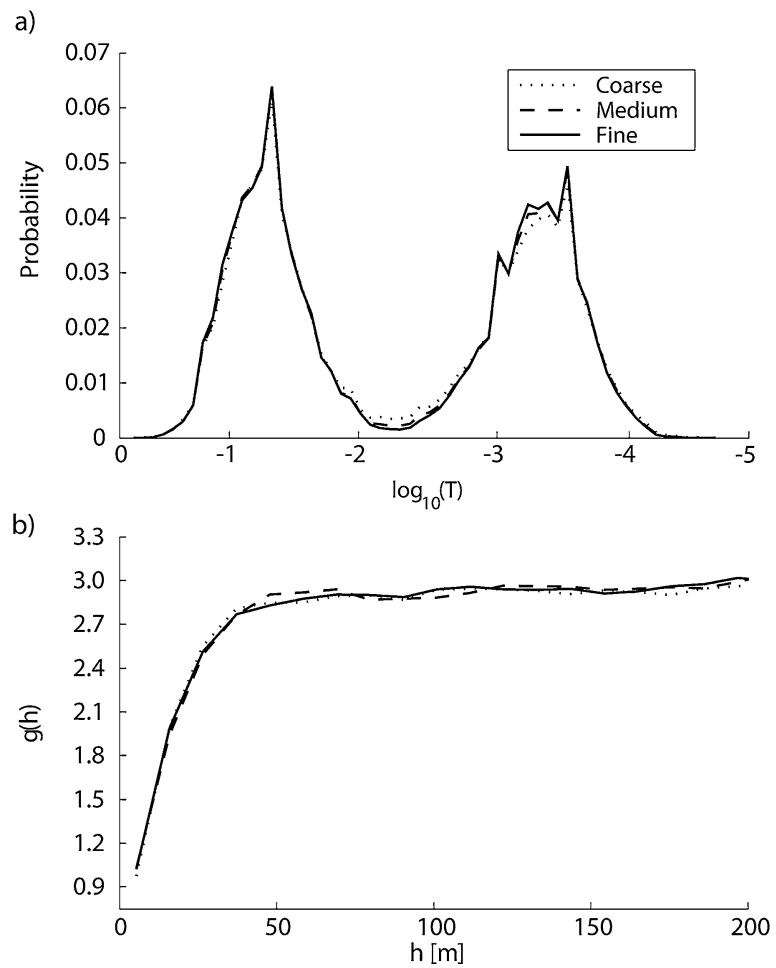

the super-resolution problem as a simulation exercise, while keeping the same spatial model for the coarse and the fine scale, therefore making an assumption of scale invariance. Other simulation techniques than MPS could be used with the same principle. However, we find that the use of MPS is especially straightforward in this case because there is no need to find or to build a training image since it is already available as the coarse image.

The resulting fine-resolution images present the same properties as their coarse counterparts, both in terms of fractal dimension and reproduction of spatial statistics. This super-resolution method is parsimonious in parameterization and valid over a wide range of scales. Fractal dimensions do not need to be calculated, nor is any adjustment of parameters necessary, except for the parameters of the multiple-point simulation algorithm. The key for inferring the fine-scale structure is the hypothesis of self-similarity applied to the patterns of the coarse image. The method does not need an explicit spatial model such as a training image or a variogram. One requirement of the method is that the coarse image should be large enough to contain a diversity of patterns to be reproduced at the fine scale. Compared to super-resolution techniques used in image processing, the proposed super-resolution method is limited to self-similar structures, which is not always the case for natural images.

The possibility to perform multivariate super-resolution mapping could be used for applications related to the estimation of fine-resolution land cover maps, based on coarse-resolution proportions of each land cover class given by remote sensing images. This could be possible because the algorithm can accommodate multivariate 
images combining both continuous and categorical variables. More generally, the method can be applied, for example, to remote sensing images whose resolution is insufficient for given applications (e.g. satellite imaging, geophysics) or time series measurements (such as precipitation intensity at a given location) that were recorded with widely spaced time steps.

Acknowledgements This work was supported by the Swiss National Science Foundation under Grants PP00P2-124979/1 and PBNEP2-124334, and the National Center for Groundwater Research and Training, Australia.

\section{References}

Arpat B, Caers J (2007) Conditional simulations with patterns. Math Geol 39(2):177-203

Atkins C, Bouman C, Allebach J (1999) Tree-based resolution synthesis. In: Image processing, image quality, image capture systems conference (PICS-99). IS\&T, Savannah, Georgia

Baker S, Kanade T (2002) Limits on super-resolution and how to break them. IEEE Trans Pattern Anal Mach Intell 24(9):1167-1183

Barton C, La Pointe P (1991) Fractals in the earth sciences. Plenum, New York

Bertero M, Boccacci P (2003) Super-resolution in computational imaging. Micron 34(6):265-273

Boucher A, Kyriakidis C, Cronkite-Ratcliff C (2008) Geostatistical solutions for super-resolution land cover mapping. IEEE Trans Geosci Remote Sens 46(1):272-283

Dai S, Han M, Xu W, Wu Y, Gong Y, Katsaggelos A (2009) SoftCuts: a soft edge smoothness prior for color image super-resolution. IEEE Trans Image Process 18(5):969-981

Deutsch C, Journel A (1992) GSLIB: geostatistical software library. Oxford Univ Press, Oxford

El Ouassini A, Saucier A, Marcotte D, Favis B (2008) A patchwork approach to stochastic simulation: a route towards the analysis of morphology in multiphase systems. Chaos Solitons Fractals 36(2008):418-436

Farsiu S, Robinson D, Elad M, Milanfar P (2004) Advances and challenges in super-resolution. Int J Imaging Syst Technol 14(2):47-57

Fattal R (2007) Image upsampling via imposed edge statistics. ACM Trans Graph 26(3): 95

Ferraris L, Gabellani S, Rebora N (2003) A comparison of stochastic models for spatial rainfall downscaling. Water Resour Res 39(12)

Freeman WT, Jones TR, Pasztor EC (2002) Example-based super-resolution. IEEE Comput Graph Appl 22(2):56-65

Grimstad A, Mannseth T, Nævdal G, Urkedal H (2003) Adaptive multiscale permeability estimation. Comput Geosci 2003(7):1-25

Guardiano F, Srivastava M (1993) Multivariate geostatistics: beyond bivariate moments. In: GeostatisticsTroia, pp 133-144. Kluwer Academic, Amsterdam

Harter T (2005) Finite-size scaling analysis of percolation in three-dimensional correlated binary Markov chain random fields. Phys Rev E 72:026120

Journel A, Zhang T (2006) The necessity of a multiple-point prior model. Math Geol 38(5):591-610

Kerrou J, Renard P, Hendricks-Franssen H-J, Lunati I (2008) Issues in characterizing heterogeneity and connectivity in non-multi-Gaussian media. Adv Water Resour 31(1):147-159

Kiraly L (1988) Large scale 3-D groundwater flow modelling in highly heterogeneous geologic medium. In: Groundwater flow and quality modelling, pp 761-775

Liu X, Coulibaly P, Evora N (2008) Comparison of data-driven methods for downscaling ensemble weather forecasts. Hydrol Earth Syst 12(2):615-624

Liu Y, Journel A (2008) A package for geostatistical integration of coarse and fine scale data. Comput Geosci 35(3):527-547

Lovejoy S, Mandelbrot B (1985) Fractal properties of rain and a fractal model. Tellus A 37:209-232

Lovejoy S, Schertzer D (1990) Multifractals, universality classes and satellite and radar measurements of cloud and rain fields. J Geophys Res 95(D3):2021-2034

Mandelbrot B (1967) How long is the coast of Britain? Statistical self-similarity and fractional dimension. Science 156(3775):636-638 
Mandelbrot B (1974) Intermittent turbulence in self-similar cascades: divergence of high moments and dimensions of the carrier. J Fluid Mech 62(2):331-358

Mariethoz G (2010) A general parallelization strategy for random path based geostatistical simulation methods. Comput Geosci 37(7):953-958

Mariethoz G, Renard P (2010) Reconstruction of incomplete data sets or images using direct sampling. Math Geosci 42(3):245-268

Mariethoz G, Renard P, Straubhaar J (2010) The direct sampling method to perform multiple-point simulations. Water Resour Res 46:W11536

Marsan D, Schertzer D, Lovejoy S (1996) Causal space-time multifractal processes: predictability and forecasting of rain felds. J Geophys Res 101(D21):333-346

McCabe M, Wood E (2006) Scale influences on the remote estimation of evapotranspiration using multiple satellite sensors. Remote Sens Environ 105(4):271-285

Mosley P (1982) Analysis of the effect of changing discharge on channel morphology and instream uses in a Braided River, Ohau River, New Zealand. Water Resour Res 18(4):800-812

Nguyen M, Atkinson P, Lewis H (2006) Superresolution mapping using a Hopfield neural network with fused images. IEEE Trans Geosci Remote Sens 44(3):736-749

Remy N, Boucher A, Wu J (2009) Applied geostatistics with SGeMS: a user's guide. Cambridge University Press, Cambridge

Renard P, de Marsily G (1997) Calculating equivalent permeability: a review. Adv Water Resour 20(56):253-278

Ronayne M, Gorelick S (2006) Effective permeability of porous media containing branching channel networks. Phys Rev E 73:026305, 1-10 pp

Sánchez-Vila X, Carrera J, Girardi JP (1996) Scale effects in transmissivity. J Hydrol 183(1-2):1-22

Schulze-Makuch D, Cherkauer D (1998) Variations in hydraulic conductivity with scale of measurement during aquifer tests in heterogeneous, porous carbonate rocks. Hydrogeol J 6:204-215

Shannon CE (1948) A mathematical theory of communication. Bell Syst Tech J 27:379-423

Shih F (2009) Image processing and mathematical morphology: fundamentals and applications. CRC Press, Boca Raton

Sroubek F, G C, J F (2007) A unified approach to superresolution and multichannel blind deconvolution. IEEE Trans Image Process 16(9)

Straubhaar J, Walgenwitz A, Renard P, Froidevaux R (2008) Optimization issues in 3D multipoint statistics simulation. In: Geostats 2008, 1-5 Dec 2008, Santiago, Chile

Straubhaar J, Renard P, Mariethoz G, Froidevaux R, Besson O (2011) An improved parallel multiple-point algorithm using a list approach. Math Geosci 43(3):305-328

Strebelle S (2002) Conditional simulation of complex geological structures using multiple-point statistics. Math Geol 34(1):1-22

Tatem A, Lewis H, Atkinson P, Nixon M (2002) Super-resolution land cover pattern prediction using a Hopfield neural network. Remote Sens Environ 79:1-14

Tsai RY, Huang TS (1984) Multiframe image restoration and registration. In: Advances in computer vision and image processing. JAI Press, Cambridge, pp 317-339

Turcotte D (1992) Fractals and chaos in geology and geophysics. Cambridge Press, Cambridge

Von Storch H, Zorita E, Cubasch U (1993) Downscaling of global climate change estimates to regional scales: an application to Iberian rainfall in wintertime. J Climate 6(6):1161-1171

Wang J, Gong Y (2008) Fast image super-resolution using connected component enhancement. In: ICME '08: IEEE international conference on multimedia and expo, June 23-26, Hannover, Germany

Wen X, Gomez-Hernandez J (1998) Numerical modeling of macrodispersion in heterogeneous media: a comparison of multi-Gaussian and non-multi-Gaussian models. J Contam Hydrol 31(1):129-156

Wen X, Deutsch C, Cullick A (1997) High resolution reservoir models integrating multiple-well production Data, SPE, SPE 38728

Wilby R, Wigley T (1997) Downscaling general circulation model output: a review of methods and limitations. Prog Phys Geogr 21(4):530-548

Wu J, Boucher A, Zhang T (2008) A SGeMS code for pattern simulation of continuous and categorical variables: FILTERSIM. Comput Geosci 34(12):1863-1876

Zlotnik VA, Zurbuchen BR, Ptak T, Teutsch G (2000) Support volume and scale effect in hydraulic conductivity: experimental aspects. In: Theory, modelling, and field investigations in hydrogeology: A special volume in honor of Shlomo P Neuman's 60th birthday. Geological Society of America, pp 215-231 